\title{
A Fully Automated Method for the Delineation of Osseous Interface in Ultrasound Images
}

\author{
Vincent Daanen ${ }^{1}$, Jerome Tonetti $^{2}$, and Jocelyne Troccaz ${ }^{1}$ \\ 1 Joseph Fourier University, \\ TIMC Laboratory, GMCAO Department \\ Institut d'Ingénierie de l'Information de Santé (IN3S) \\ Faculty of Medecine - 38706 La Tronche cedex - France \\ ${ }^{2}$ University Hospital \\ Orthopaedic Surgery Department \\ CHU A Michallon, BP217 \\ 38043 Grenoble
}

\begin{abstract}
We present a new method for delineating the osseous interface in ultrasound images. Automatic segmentation of the bone-soft tissues interface is achieved by mimicking the reasoning of the expert in charge of the manual segmentation. Information are modeled and fused by the use of fuzzy logic and the accurate delineation is then performed by using general a priori knowledge about osseous interface and ultrasound imaging physics. Results of the automatic segmentation are compared with the manual segmentation of an expert.
\end{abstract}

\section{Introduction}

In computer-aided orthopaedic surgery (CAOS), the knowledge of the bone volume position and geometry in the operative room is essential. The usual way to acquire it is to register pre-operative data with intra-operative data. For the last years, the use of ultrasound imaging as intra-operative imaging has significantly increased ( $\left.\left.\begin{array}{llll}1 & 2 & 3 & 4\end{array}\right]\right)$ because such imaging investigations are inexpensive and riskless ; and using 6D localized ultrasound probe makes it possible to reconstruct the 3D shape of a structure after its delineation. The extraction of structures from ultrasound data appears to be a delicate key point in CAOS. Several automatic or semi-automatic methods have been proposed to achieve the delineation of features in ultrasound images but these methods apply to specific parts of the human body ([2] $)$ because of the poor quality of the ultrasound images (low contrast, low signal-to-noise ratio and speckle noise). We propose a fully automated method in order to achieve the delineation of the bone-soft tissues interface in ultrasound images based on data fusion : data available in images are modeled and fused by the use of fuzzy logic. We then mimic the expert's reasoning to accurately delineate the osseous interface. 


\section{Material and Method}

\subsection{Material}

Ultrasound imaging is achieved using a linear US probe, $25 \mathrm{~mm}$ large, working at a frequency of $7.5 \mathrm{MHz}$. The probe is localized in $3 \mathrm{D}$ space by an optical localizer. The US probe is calibrated according to the technique described in [5] (the pixel size is about $0.1 \mathrm{~mm} /$ pixel). The position of an image pixel is known in $3 \mathrm{D}$ space with a precision in the range of the optical localizer (i.e. $1 \mathrm{~mm}$ ).

\subsection{Method}

In this section, we introduce the expert's reasoning and the way we mimic it in order to achieve an accurate segmentation of the osseous interface.

Expert's reasoning. The expert's reasoning is based on one hand on the physics of the ultrasound imaging and on the other hand on his knowledge of anatomy. Five properties can be pointed out :

1 - bones appear to be hyper-echoic

the great difference of acoustical impedance between bones and surrounding soft-tissues generates an important echo.

2 - bones are said to 'stop' ultrasound waves

this is due to the high absorption rate of bones.

3 - the reflection is almost completely specular

only interfaces almost perpendicular to the direction of the ultrasound beam will reflect so features of interest appear to be composed of horizontal (or near horizontal) parts.

4 - non-broken bone surface do not present major discontinuities the found osseous interface should be as smooth as possible.

5 - among an osseous interface, the contrast appears to be homogeneous

We propose a method that models these information and mimics the reasoning of the expert. The method is divided into 3 steps :

- the image processing step

which aims at modeling the information available in the images and then concentrate them into one image representing the membership of the pixel to a given property. This stage models and fuses points 1,2 and 3 cited above.

- the 'continuity-ness' cost function computation step

which aims at finding continuous osseous interfaces from the fuzzy image. We model here the point 4.

- the contrast computation and decision making

which purpose is to select the optimal osseous interface from the candidates at the previous step from the fifth property. 


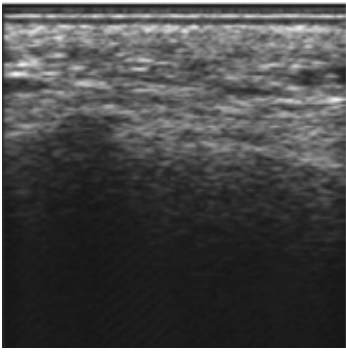

(a) ultrasound image

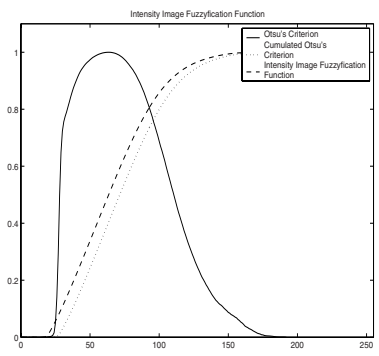

(b) intensity fuzzification function

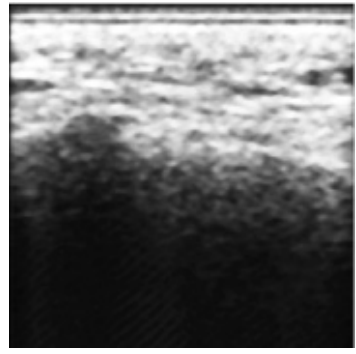

(c) fuzzy intensity image

Fig. 1. Computation of FII from the original ultrasound image (pelvic bone)

\section{Image Processing Step}

Fuzzy Intensity Image: this image attempts to model the first property, i.e. in ultrasound imaging, bones appear as hyper-echoic and therefore bright pixels constitute an indication of the location of the osseous interface. In a previous development [6], we pointed out that binarizing the initial ultrasound image using the Otsu's threshold ( $T_{\text {Otsu }}$ ) gives a good approximation of the echogenic area and so, of the position of the osseous interface.

We make use of this information to build the fuzzification function $\mu_{\text {Int }}$ : the criterion (we call $V_{\text {Otsu }}$ : Fig[1-b, solid curve), needed to compute $T_{O t s u}$, is used as follows ; first, $V_{\text {Otsu }}$ is normalized and cumulated (Fig 1 1 b, dotted curve) and it is then shifted in order to force the membership function value :

$$
\mu_{\text {Int }}\left(T_{\text {Otsu }}\right)=0.5
$$

The fuzzification function (Fig 1 b b, dashed curve) is finally applied over the graylevel image in order to achieve the construction of the fuzzy intensity image $F I I(p)$ which gives for a pixel $p$ of the intensity image its membership degree to the echogenic area.

Fig.1 a shows an ultrasound imag 1 of the sacrum part, the intensity fuzzification function and the Intensity Fuzzy Image (Fig,1 $\mathrm{f}$ ).

Fuzzy Gradient Image: the gradient information constitutes another important part in the determination of the osseous interface and so the fuzzy gradient image $F G I(p)$ is of great interest. Indeed, the transition from the bone to the acoustic shadow area suggests to search for highly contrasted pixels (properties 1 and 2) and because ultrasound imaging should only 'detect' structure changes which are perpendicular to the ultrasound beam (point 3: Fig. 2 $\mathrm{ra}$ ), we make use a $5 \times 5$ 'horizontal-direction' MDIF edge detector, which is a first-order derivative filter obtained by the convolution of the 4 -connexity $3 \times 3$ mean lowpass filter with the Prewitt's derivative kernels.

\footnotetext{
${ }^{1}$ All the images have been cropped to half their length because the inferior part does not present any interest
} 


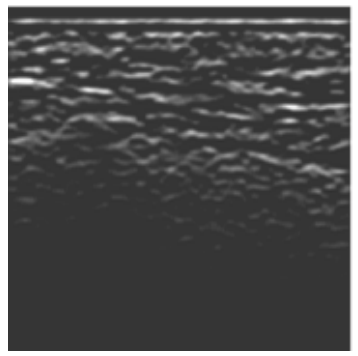

(a) gradient image of image Fig 1 a

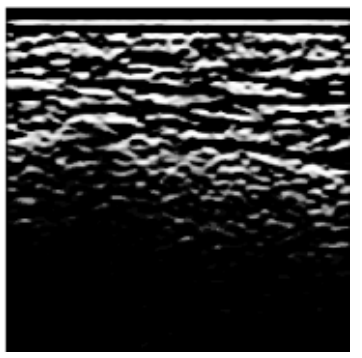

(b) fuzzy gradient image

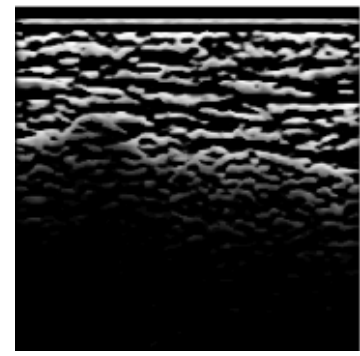

(c) data fusion image

Fig. 2. Gradient image, FGI and Fusion Image of the original ultrasound image

The resulting image is then thresholded in order to remove the transition from dark-to-bright. Finally, we use the S-shape function to perform the fuzzification of the gradient image and obtain the Fuzzy Image Gradient $F G I(p)$ (Fig. $2 \mathrm{~b}$ ). The parameters of the S-shape function are computed such that $S(x)$ is the closest s-shape function to the normalized cumulative histogram.

Data Fusion: a pixel of the US image may belong to the osseous interface if both its gray-level and gradient are 'high'. This combination is achieved by the use of the 'conjunctive-type' combination operator min . The membership of a pixel to the osseous interface is then given by :

$$
F I(p)=\min (F I I(p), F G I(p))
$$

$F I(p)$ denotes the global degree of membership of the pixel $p$ to the echogenic area and to an highly contrasted image area (Fig.27c).

Determination of the osseous interface. According to the expert's reasoning, the optimal threshold described a continuous interface where the local contrast is maximum and homogeneous. For each membership degree $0<\mu<1$ ( $\mu$ space is discretized with a step $\delta_{\mu}=0.005$ ), the defuzzification of $F I(p)$ is performed and the 'continuity-ness' of the profile is evaluated. We then choose the membership degree which maximizes the local contrast and its homogeneity, and also ensures a local maximum continuity of the profile.

Defuzzification at a given membership degree $\mu$ : the defuzzification process aims at extracting from the fuzzy image $F I(p)$ the osseous interface related to a membership degree $\mu_{\text {ref }}$. To achieve this task, we make use of a priori knowledge about the physics of ultrasound imaging. As mentioned earlier, bones 'stop' the US-waves and so, for a column of the image, the pixel of the osseous interface related to a membership value $\mu_{\text {ref }}$ is the last (from the top) pixel which has a membership equal to $\mu_{\text {ref }}$. At the end of this defuzzification process, at the most one pixel per column is highlighted. The 'curve' described by these pixels is called profile in the rest of the paper. 


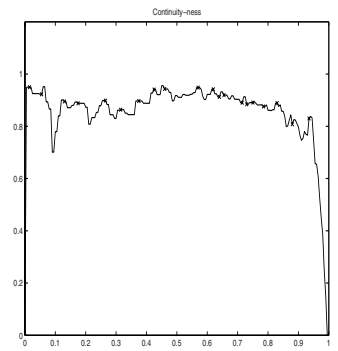

(a) 'Continuity-ness'

objective function

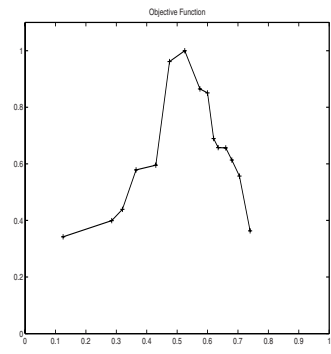

(b) Continuity and

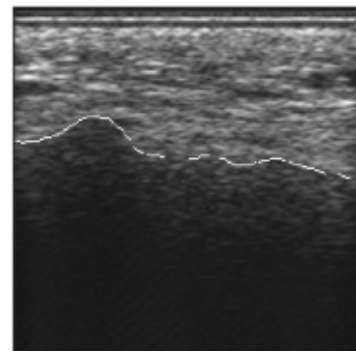

(c) resulting segmentation image

homogeneity objective function

Fig. 3. Computation of FII from the original ultrasound image

Evaluation of the 'continuity-ness' of the profile: because, actual osseous interfaces do not present discontinuities, the osseous interface we detect should be as smooth as possible. We use this property to determine the optimal defuzzification threshold by computing a function that reflects the 'continuity-ness' of a computed osseous interface.

The measure of the 'continuity-ness' of a profile is achieved by applying the wavelet transform to it : the wavelet transform decomposes the profile with a multiresolution scale factor of two providing one low-resolution approximation $\left(A_{1}\right)$ and one wavelet detail $\left(D_{1}\right)$. We then apply the wavelet transform to $A_{1}$ and get a second order low-resolution approximation $\left(A_{2}\right)$ and wavelet detail $\left(D_{2}\right)$. The Detail signals are then used to quantify the discontinuities of the original profile. The 'amount' of discontinuities in the profile is computed as follows :

$$
\varepsilon(\mu)=E\left(D_{1}\right)+E\left(D_{2}\right)+P e n
$$

where $E(s)$ is the energy of a signal $s(t)$.

Experimentally, we choose the Daubechies-4 wavelet basis (several other bases have been tested and no dependence was pointed out at the exception of the Haar Basis). Pen is a penalization term related to the length of the profile which is used to reject small osseous interfaces detected when $\mu_{\text {ref }}$ is unsuitable (i.e. too high).

Finally, $\varepsilon(\mu)$ is normalized (giving $\varepsilon^{\prime}(\mu)$ ) and we compute the 'continuity-ness' of the profile as :

$$
C(\mu)=1-\varepsilon^{\prime}(\mu)
$$

As one can see (Fig[3a), the 'continuity-ness' function $C(\mu)$ presents several local maxima. Each of them locates a membership degree $\mu$ where the associated profile is more continuous than the profiles of its neighbors and so each of them may be the optimal defuzzification threshold. We detect them by computing the watershed transform of $C(\mu)$. For each local maxima, the image is defuzzed to the corresponding membership degree $\mu$ and the local contrast is computed. 
Local contrast computation: for each pixel $p$ belonging to a profile, the local contrast $L C(p)$ associated to the pixel $p$ is computed by :

$$
L C(p)=\frac{\overline{U p}-\overline{\text { Down }}}{\overline{U p}+\overline{D o w n}}
$$

where $\overline{U p}$ (resp. $\overline{D o w n}$ ) is the mean value of the above (resp. underneath) regionof -interest2. The global contrast along the profile Contrast $(\mu)$ is then computed by :

$$
\operatorname{Contrast}(\mu)=\sum_{p} L C(p)
$$

We also evaluate the homogeneity of the local contrasts along a profile by computing the standard deviation of the values along it. This gives us a function $\operatorname{StdDev}(\mu)$.

Optimal defuzzification threshold determination: Finally, the optimal membership degree is the one that maximized $\operatorname{Cost}(\mu)$ :

$$
\left.\mu_{\text {Optimal }}=\arg \max \operatorname{Cost}(\mu)\right)
$$

where

$$
\operatorname{Cost}(\mu)=\operatorname{Contrast}(\mu)+\frac{1}{\operatorname{StdDev}(\mu)}
$$

Fig 3 b shows the objective function computed by (8) and the resulting segmentation is shown in Fig 3 c.

\section{Results}

The proposed method has been tested on ultrasound images of sacrum coming from cadaver datasets or patient datasets : about 300 images have been processed. For each image, the manual segmentation of the expert is available and constitutes our bronze-standard.

For each image within a dataset, we compute the error between the manual segmentation and the segmentation computed by our method. We then compute the mean error for each image (Table 1 fcolumn 1) and the Hausdorff distance and mean absolute distance (average of all the maximum errors within a subset) (Table 1 f column 2). In order to evaluate the ability of the proposed method to delineate the osseous interface in strongly corrupted images, we also compute the Signal-to-Mean Squared Error ratio (Table 1 column 3)(see [7] for more information about the $S / M S E)$.

\footnotetext{
${ }^{2}$ A roi is an area of 10 pixels long-by-1 pixel large
} 
Table 1. Segmentation differences

\begin{tabular}{|c|c|c|c|}
\hline & $\begin{array}{c}\text { Segmentation } \\
\text { Error }\end{array}$ & Max Errors & S/mse \\
\cline { 2 - 4 } Dataset & mean/SD (pixel) & mean/max (pixel) & mean/SD (dB) \\
\hline Patient 1 (51 images) & $7.808 / 1.995$ & $12.137 / 22$ & $5.052 / 0.185$ \\
\hline Patient 2 (49 images) & $8.807 / 3.177$ & $16.905 / 25$ & $5.206 / 0.428$ \\
\hline Patient 3 (69 images) & $4.545 / 3.874$ & $17.0789 / 35$ & $8.905 / 0.283$ \\
\hline Cadaver 1 (37 images) & $3.495 / 1.931$ & $9.830 / 36$ & $8.786 / 0.340$ \\
\hline Cadaver 2 (41 images) & $2.679 / 1.456$ & $7.294 / 19$ & $9.019 / 0.259$ \\
\hline Cadaver 3 (39 images) & $4.056 / 3.213$ & $12.14 / 38$ & $7.984 / 0.177$ \\
\hline
\end{tabular}

pixel size is $0.112 \mathrm{~mm} x 0.109 \mathrm{~mm}$

As one can see (table 1), as compared to the manual delineation of the expert:

- the mean error of segmentation is always less than 10 pixels (i.e. $1 \mathrm{~mm}$ ) even on highly corrupted images. However, it is clear that the accuracy of the delineation is correlated within the amount of noise and therefore, we think that taking into account the noise (measured by the $\mathrm{S} /$ sme ratio by example) during the fusion and/or delineation process may be a way to improve the delineation.

- the maximum errors still remain important but, according to us, it is not the error we should focus on : we point out that these errors occur at more or less one pixel on complex shapes (such as medial sacral crest or sacral hiatus) giving thus an important maximum error comparatively the manual delineation but the overall error on the global shape still remains negligible and has very limited impact on the registration step which follows.

- the proposed method is also sufficiently fast to be used during the intra-operative stage of a CAOS : the time needed to delineate one image is less than $4 \mathrm{~s}$ on a Pentium $I I I-800 \mathrm{MHz}$ PC. The processing of large datasets such as Patient 3 takes about 4 minutes whereas it would take more than 30 minutes in the case of a manual delineation (according to [1]).

This method have also been used to delineate the osseous interface in femur and vertebrae ultrasound images and the osseous interface was well delineated in particular the spinous process in vertebrae images.

\section{Discussion}

Recently, lots of methods dedicated to the indirect delineation of the bone surface in ultrasound images have been proposed in the literature ([2] ) but these methods have not been tested on real patients' datasets yet. Moveover, the ultrasound imaging is constrained by the use of a mechanical system ([2]); and a good initial estimation of the rigid registration matrix between the CT and US datasets is often required to achieve the bone surface segmentation in the ultrasound images $(\underline{3,4})$. 
The method described in this paper does not require neither a dedicated ultrasound images acquisition system nor an estimation of the rigid registration matrix between the CT and US datasets to perform the delineation of the osseous interface in ultrasound images. Moreover, it has been extensively tested on images acquired on cadavers (about 120 images) and on real patients (about 170 images). We point out that although the method is sensible to noise, the mean errors are still acceptable : we measure a maximum mean error of 8.8 pixels (i.e. $0.8 \mathrm{~mm}$ ) with a $S / m$ se ratio of $5.206 \mathrm{~dB}$ which corresponds to a highly corrupted image (according to [7).

We do not notice any dependence of the results to the visualization parameters tuning at the exception that the osseous interface should not get bogged down in noise. We think that this condition is acceptable since the physician has to validate the images during the acquisition stage (and so, this validation can only be done if he is able to localize the osseous interface).

We think that an important point has also to be made clear : the validation, based on the comparison to a single expert segmentation, may appear limited. However, segmenting bones on ultrasound images is very unusual for physicians and it is difficult to find several expert users. Moreover, gold-standard does not exist and tests on phantoms or isolated bones would not allow to draw conclusions applicable to real data. Thus, we consider that this evaluation is a first step.

\section{Conclusion}

In this paper, we presented a method for automatic delineation of the osseous interface in ultrasound image. The method is based on the fusion of the pixels intensity and gradient properties in a first step and then on the fusion of information extracted from the physics of ultrasound imaging and a priori knowledge. The method has been used to delineate osseous interface in ultrasound images of the sacrum which may present several shapes ; we also use it to delineate the osseous interface in vertebrae images and good results were obtained in all cases. As it is independent of the shape to be recovered we think that the described method is a first step toward robust delineation of the osseous interface in ultrasound images.

\section{References}

1. L. Carrat, J.Tonetti, S. Lavallée, Ph. Merloz, L. Pittet, and JP. Chirosset. Treatment of Pelvic Ring Fractures : Percutaneous Computer Assisted Iliosacral Screwing. In CS Serie, Springer Verlag, MICCAI, volume 1496, pages 84-91, 1998.

2. B. Brendel, S. Winter, A. Rick, M. Stockheim, and H. Ermert. Registration of 3D CT and Ultrasound Datasets of the Spine using Bone Structures. Computer Aided Surgery, 7(3):146-155, 2002.

3. D.V. Amin, T Kanade, A.M. DiGioia III, and B. Jaramaz. Ultrasound Registration of the Bone Surface for Surgical Navigation. Computer Aided Surgery, (8):1-16, 2003. 
4. G. Ionescu, S.Lavallée, and J. Demongeot. Automated Registration of Ultrasound with CT Images : Application to Computer Assisted Prostate Radiotherapy and Orthopedics. In CS Serie, Springer Verlag, MICCAI, volume 1679, pages 768-777, 1999.

5. T. Lang . Ultrasound Guided Surgery : Image Processing and Navigation. PhD thesis, Norwegian University of Science and Technology, Trondheim, Norway, 2000.

6. V. Daanen, J. Tonetti, J. Troccaz, and Ph. Merloz. Automatic Determination of the Bone-Soft Tissues Interface in Ultrasound Images. First Results in Iliosacral Screwing Surgery. In Proceedings of Surgetica-CAMI 2002, pages 144-151, 2002.

7. A. Achim, A. Bezerianos, and P. Tsakalides. Novel Bayesian Multiscale Method for Speckle Removal in Medical Ultrasound Images. IEEE Transactions on Medical Imaging, 20(8):772-783, 2001. 